\title{
The Hydrogen-Metal Interaction
}

\author{
J.K. Nørskov, Lyngby \\ (Laboratory of Applied Physics II, Technical University of Denmark)
}

Hydrogen interacting with metals attracts more attention than any other gas-solid system. There are a number of good reasons why this is so. Hydrogen has a solubility which is higher than that of most other gases. This makes hydrogen-metal systems very attractive for experimental studies of the properties of impurities in metals. Once an impurity has been introduced into a solid, translational symmetry is broken and completely new theoretical tools must be introduced. Hydrogen is the simplest impurity to study theoretically because of its lack of core electron structure. The hydrogen-metal system is therefore often used as the testing ground for new techniques. Hydrogen also exhibits unique quantum behaviour when inside and adsorbed on metals. At low temperatures, tunnelling becomes a possible migration mechanism, and the indications are that the hydrogen ground state wave function is rather delocalized. At higher temperatures the diffusion is dominated by classical activated hopping. When de-
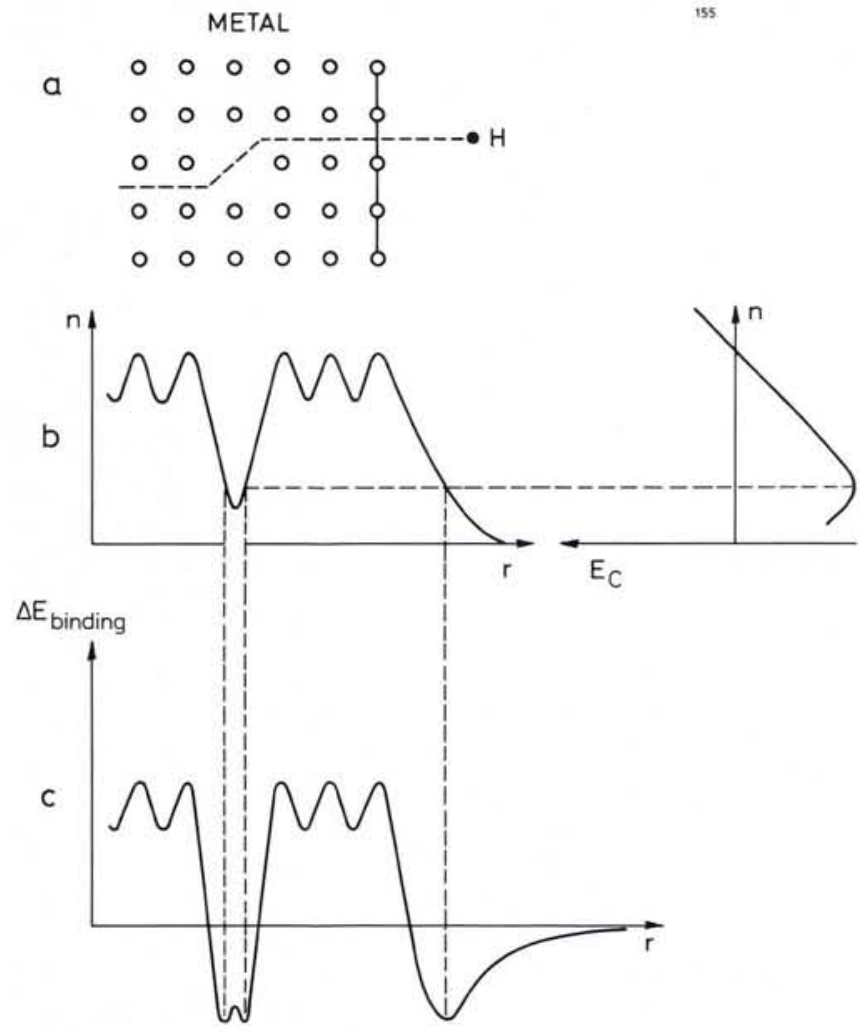

Fig. 1 - Illustration of the way the hydrogen binding energy is constructed from the metal electron density using the energy function $\mathrm{E}_{\mathrm{c}}(\mathrm{n})$. The electron density (b) and hydrogen binding energy $(c)$ is shown along the path indicated in (a). qualitatively and semi-quantitatively for most of the observed phenomena.

\section{The Hydrogen-Metal Interaction}

The basis for describing an atom interacting with a solid must be an understanding of the adiabatic potential energy surface as a function of the atom and all the solid nuclear coordinates. This is, of course, an impossible task in general, but simplified total energy calculations supported by large scale computations for particular, high symmetry systems [1] have given a very powerful picture of the interaction, which enables us to describe theoretically a large part of the complex phenomena we are studying.

The key concept that must be introduced for this purpose is the energy function $E_{c, i}(n)$ describing the energy of an atom of type $i$ in a homogeneous electron gas of density $n$. Using the variational principle of density functional theory it can be shown [2] that the total energy of a system of $N$ interacting atoms can be written:

$$
E_{\text {tot }}=\sum_{i=1}^{N} E_{c, i}\left(\overline{n_{i}}\right)+E_{\text {corr }}
$$

The first term can be thought of as describing the average effect of the surroundings on each of the atoms $i$. The density argument $\bar{n}_{i}$ in the $E_{c, i}$ function is the average of the electron density from the neighbouring cells, and $E_{c, i}\left(\overline{n_{i}}\right)$ is the binding energy of atom $i$ in the average surroundings'. The corrections $E_{\text {corr }}$ to this come from the deviations of the true surroundings from homogeneity. They can also be evaluated. They are smallest when the surroundings are as close to those of a homogeneous electron gas as possible. This is true for metals in general and the simple metals in particular. For the transition metals, $E_{\text {corr }}$ includes any direct interaction with the $d$ electrons.

There is one $E_{c}$ function for each atom, describing, to a first approximation, the bonding of this atom in any environment. It can be calculated once and for all, at least under certain local approximations to treat electronic exchange and correlation effects. Except for the inert gases the $E_{c}$ functions 


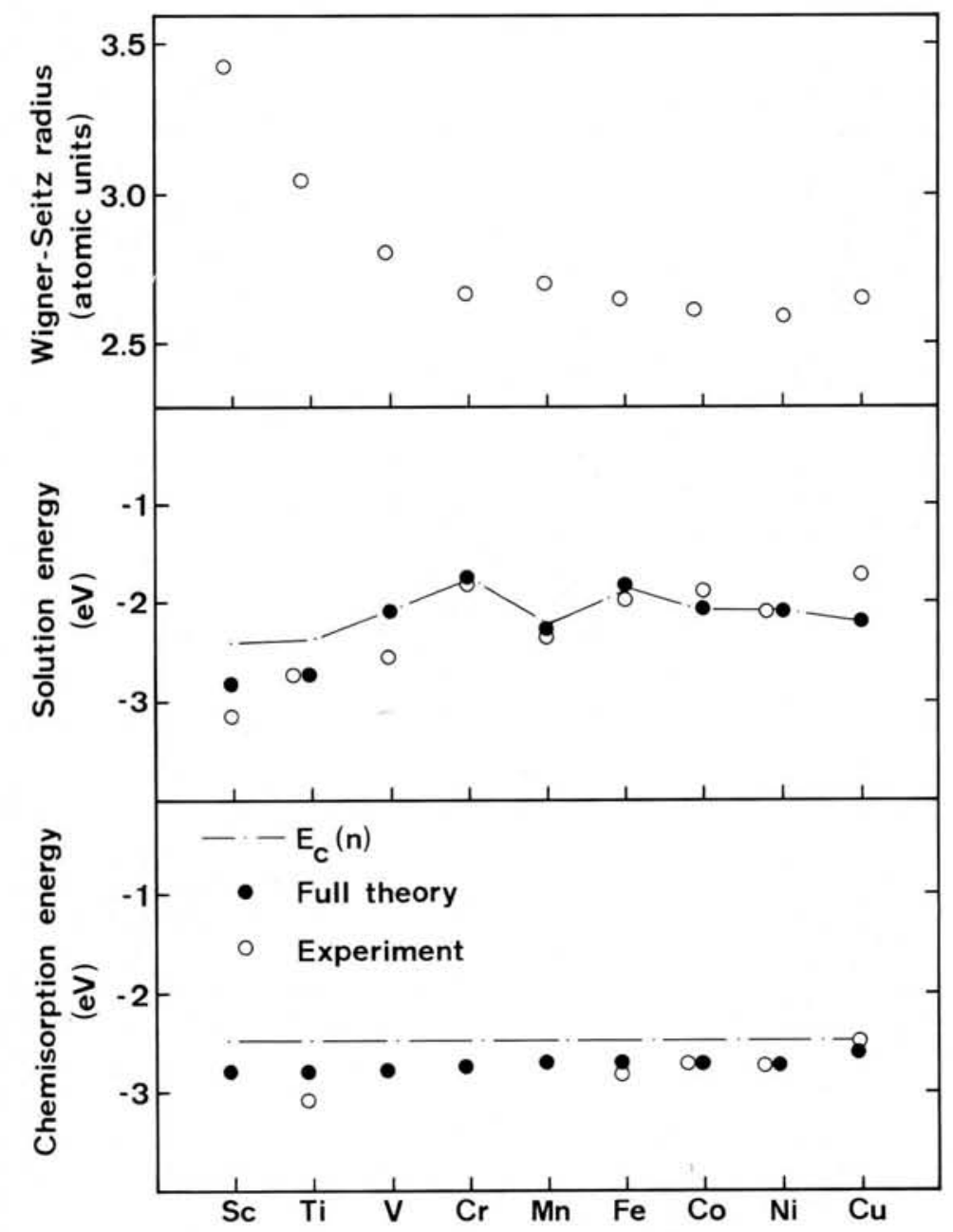

Fig. 2 - Comparison of theoretical and experimental binding energies for hydrogen inside and outside the $3 d$ transition metals. Both the contribution from the density dependent term $\mathrm{E}_{\mathrm{c}}(\mathrm{n})$ of Eq. (1) and the result of the full calculation including the correction terms are shown.

show a single minimum, indicating that there is an optimum surrounding density, where the atom in question binds with an energy given by the depth of the minimum.

The $E_{c}$ function alone is capable of explaining qualitatively the observed behaviour of hydrogen on and inside a metal. This is illustrated schematically in Fig. 1. In order to estimate the energy of a hydrogen atom as it passes into and through a metal, we need an idea of the metal electron density variations. This is illustrated in Fig. 1(b) for the hydrogen path shown in Fig. 1(a). Outside the surface, the electron density falls off exponentially to zero. Inside the crystal, the density is highest at points close to one or more metal atoms, and smallest in the most open sites in the lattice, the interstitial octahedral site in the hcp and fcc structures and the interstitial tetrahedral site in the bcc structure. Open defects like a vacancy lead to a considerably smaller electron density towards the centre. Given the $E_{c}$ function for hydrogen the potential energy variations can then be constructed as shown in Fig. 1(c). As the hydrogen atom approaches the surface it will encounter the optimum density at some point. This gives rise to the chemisorption minimum in which the atom binds outside the surface. This is usually the most stable site of all because the density is higher than optimum everywhere else in the lattice. The chemisorption bond length is given to a first approximation by the requirement that the metal electron density be equal to the optimum.

Moving further into the lattice the density rises and the $E_{c}$ function becomes almost linear in density. The energy variations are therefore a direct reflection of the variations in the metal electron density. The sites of lowest energy will be the most open sites, octahedral in fcc and hcp and tetrahedral in bcc. The activation energy reflects the minimum density change needed to move from one site to the next. This is highest in the close-packed fcc and hcp structures; diffusion is thus fastest in the bcc metals.

Any open defect will act as a trap for hydrogen through lowering the energy by an amount proportional to the decrease in electron density. The more open the defect, the larger the trapping energy, which therefore increases in the sequence: dislocation $\leq$ vacancy $\leq$ void. At the centre of a vacancy, the density may be less than the optimum and the hydrogen atom then does not occupy the centre site.

One more thing is immediately clear from the discussion above. Because the interstitial electron density in most metals is larger than optimum for hydrogen, interstitial hydrogen impurities will induce a local expansion of the surrounding lattice. This is very important for the diffusion process, which then involves motion not only of the hydrogen, but of a number of metal coordinates as well.

The quantitative applicability of the concept of a unique energy vs. density function describing the energetics of an atom like hydrogen in various different surroundings is illustrated in Fig. 2. Here the hydrogen binding energy inside and outside the $3 d$ transition metals are compared to values deduced from experiments. The simple estimate based on the interstitial electron density and the $E_{c}$ function correctly describes the trends in the heat of solution. The trend is a reflection of the variations in the interstitial electron density of the metal. The smaller the lattice constant (or Wigner-Seitz radius) the higher the density and the higher the hydrogen energy. The uniquely high stability of hydrogen in Pd is thus a reflection of a very low interstitial electron density in Pd. This, in turn, is a consequence of a lattice constant, which is very large compared to the 'size' of the atom, or, if you like, to the relatively weak Pd-Pd bonds.

Outside the surface, the hydrogen atom can always find a position with the optimum electron density. ConsequentIy, the $E_{c}$ function gives a constant contribution to the chemisorption energy. The trends are here given by the variation in the $\mathrm{H}$ 1s-metal d interaction, which increases as the $\mathrm{d}$ - band empties towards the left in the transition metal series. This is included in the correction terms in Eq. (1). The result of the full calculation, including the extra 1s-d hybridization, is also included in the figure.

\section{Hydrogen Diffusion}

A detailed understanding of the dynamics of hydrogen on the adiabatic potential energy surface is still far away. It is clear that the diffusion properties vary considerably with temperature [3]. This is indicated schematically in Fig. 3.

At the lowest temperature, coherent tunnelling will dominate. The hydrogen 


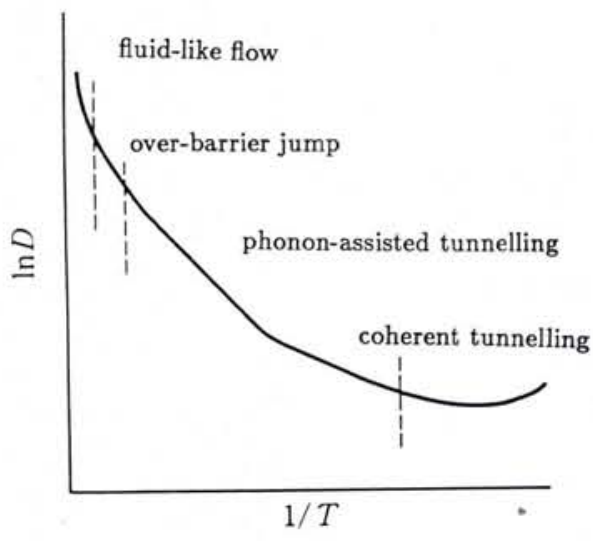

Fig. 3-Schematic illustration of the variation of the hydrogen diffusion constant with temperature. Various regimes can be distinguished as discussed in the text. From Ref. 2.

atoms will move in Bloch states extending over many unit cells [4]. The diffusion rate will be given by the band-width of the hydrogen bands. The non-adiabatic coupling to the metal electron-hole pair excitations gives rise to a renormalization of the band-width calculated from the adiabatic potential energy surface [5]. This renormalization can be described as a consequence of the "Anderson orthogonality catastrophe", namely: the conduction electron eigenstate with the particle perturbed at one site has an infinitesimal overlap with the eigenstate with the particle in another site. Hopping therefore involves infinitesimal electronhole pair excitations and is suppressed.

As the temperature is increased, phonon assisted tunnelling becomes possible [3]. The thermal motion of the lattice may produce a configuration where the tunnelling matrix element is larger than that of the undistorted lattice. At the same time, the tunnelling becomes incoherent owing to the fluctuations of the phonons. The same thing happens with fluctuations in the electron system at an even lower temperature, because the coupling to the electrons is stronger [6]. The result is that the life-time of the Bloch states decreases with increasing temperature. When the temperature becomes high enough for the coherence time $\tau$ to be shorter than the average time between jumps, the motion is completely diffusive with a diffusion constant [6]

$$
D \cong W_{\text {ren }}^{2} \tau a^{2}
$$

where $W_{\text {ren }}$ is the renormalized band width, and $a$ is the lattice constant.

At even higher temperatures, the hydrogen atoms behave completely classically moving through the lattice by thermally activated over-barrier jumps. Hydrogen is so heavy and the barriers for diffusion so large that this is the dominant migration mechanism for most systems at most temperatures. When defects are present, the trapping and detrapping of hydrogen can dominate the diffusion properties [7]. Typical trapping energies are of the order $0.5 \mathrm{eV}$ (compare the interstitial- and chemisorption energies in Fig. 2). Defects can therefore increase the apparent activation energy for diffusion considerably.

Crossover to the tunnelling regime has been observed at low temperatures in three cases [2]:

1) for hydrogen in bcc metals,

2) for chemisorbed hydrogen, and

3) for the very light isotope $\mu^{+}$in fcc metals.

The first two cases are characterized by a low (adiabatic) barrier for diffusion. As discussed above, the barrier is considerably smaller in the open bcc structure than in the more close-packed structures and, outside the surface, the barrier is very small for any structure, because the hydrogen atom can find the optimum density anywhere in the surface unit cell. The last case is characterized by the small mass of $\mu^{+}$, making all quantum effects more pronounced. Quantum delocalization of hydrogen has been observed for hydrogen trapped in vacancies in $\mathrm{Ni}$ and $\mathrm{Pd}$ using channelling techniques at very low temperatures. By increasing the temperature, the occupation of excited states with a drastically different density distribution can be detected. The excitation energy involved is only of the order $3 \mathrm{meV}[8]$.

At the highest temperatures, the motion of the hydrogen will be liquid-like.

\section{Summary}

The extensive experimental and theoretical studies of hydrogen inside and on metals have resulted in a convincing physical picture of the interaction. Binding sites, diffusion barriers, lattice relaxations, interactions with lattice defects, and trends in binding energies from one metal to another can be understood within a simple theoretical model.

In the process, a new description of bonding in a metallic medium has been developed, based on the concept of a unique function relating the energy of a given atom to the surrounding electron density. The theoretical framework, the effective medium theory [8], is capable of describing a vast number of systems and now forms the basis for classical simulations of temperature- and timedependent properties. The hydrogen embrittlement process has, for instance, been simulated in this way [9].

Particular interest is focussed on the hydrogen - metal system because it provides a unique possibility for studying the quantum mechanics of a particle in contact with a heat bath. The theoretical and experimental development is fast. Quantum diffusion has now been established experimentally for a number of systems and theoretically, new insight has been gained into the coupling of the hydrogen motion to both phonons and electron-hole pair excitations. This opens the door to a new detailed understanding of hydrogen dynamics.

\section{REFERENCES}

[1] Feibelman P. and Hamann D.R., Surf. Sci. 182 (1987) 411; Hamann D.R., J. Electron Spec. \& Rel. Phen. 44 (1987) 1.

[2] Jacobsen K.W., Nørskov J.K. and Puska M.J., Phys. Rev. B 35 (1987) 7423.

[3] Fukai Y. and Sugimoto H., Adv. Phys. 34 (1985) 263.

[4] Puska M.J., Nieminen R.M., Manninen M., Chakraborty B., Holloway S. and Nørskov J.K., Phys. Rev. Lett. 51 (1983) 1081.

[5] Kondo J., Physica B 124 (1984) 25.

[6] Hedegård P., Phys. Rev. B 35 (1987) 6127.

[7] Myers S.M., Nordlander P., Besenbacher F. and Nørskov J.K., Phys. Rev. B 33 (1986) 854.

[8] Besenbacher F., Nørskov J.K., Puska M.J. and Holloway S., Phys. Rev. Lett. 55 (1985) 852

[9] Daw M.S. and Baskes M.I., Phys. Rev. Lett. 50 (1983) 1285.

\section{Meetings}

\section{Up-date and Corrections}

\section{Address omitted in March}

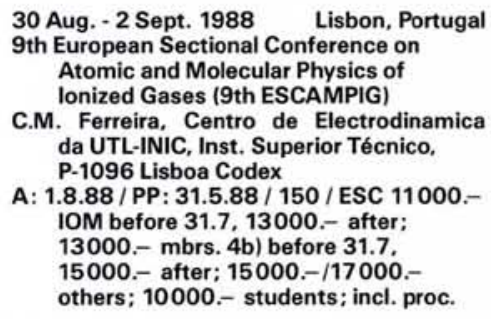

C.M. Ferreira, Centro de Electrodinamica da UTL-INIC, Inst. Superior Técnico. P-1096 Lisboa Codex

A: $1.8 .88 /$ PP: $31.5 .88 / 150 /$ ESC 11000.IOM before 31.7, 13000.- after: 13000.- mbrs. 4b) before 31.7 . 15000.- after: $15000 .-/ 17000$.others; 10000.- students; incl. proc.

\section{New}

5-9 Sept. 1988 Liège, Belgium

Workshop on the Future of Small-gap II-VISemiconductors

R. Evrard, Institut de Physique,

B-4000 Sart Tilman, Liège 1

NP / 40 / inv. / NATO / FB 10000. - all incl.

\section{New}

19-23 Sept. 1988 Restructuring of Physical Sciences in Europe and the USA, 1945-60

E. Di Silvestro, Dipto. di Fisica, Università di Roma 'La Sapienza', Ple. A. Moro, I-00185 Roma A: 15.6.88/PP / LIT 100000. - incl. proc

\section{Replaces School in Erice announced for} 3-4 November 1988

2-6 Nov. 1988 Erice, Italy

Int. School of Materials Sci, and Technol. Workshop: Point, Extended and Surface Defects in Semiconductors

A. Cavallini, Dip. di Fisica, Via Imerio 46, 1-40124 Bologna

or G. Benedek, Dip. di Fisica, Via Celoria 16 I-20133 Milano 